\title{
Author Correction: MCT2 mediates concentration-dependent inhibition of glutamine metabolism by MOG
}

Louise Fets (D), Paul C. Driscoll (D), Fiona Grimm (D), Aakriti Jain (D), Patrícia M. Nunes, Michalis Gounis (D), Ginevra Doglioni, George Papageorgiou, Timothy J. Ragan, Sebastien Campos, Mariana Silva dos Santos (D), James I. MacRae (D), Nicola O'Reilly (iD, Alan J. Wright, Cyril H. Benes, Kevin D. Courtney, David House (iD and Dimitrios Anastasiou (D)

Correction to: Nature Chemical Biology, https://doi.org/10.1038/s41589-018-0136-y, published online 8 October 2018.

In the version of this article originally published, funding information for co-author Aakriti Jain was missing. A sentence has been added to the Acknowledgements section, stating "Work by A.J. was supported by the MetaRNA Marie Skłodowska-Curie Innovative Training Nerwork (642738)." The error has been corrected in the HTML and PDF versions of the paper.

Published online: 23 October 2019

https://doi.org/10.1038/s41589-019-0409-0

(c) Springer Nature America, Inc. 2019 